\title{
Concept for parallel placement of flexible needles for Irreversible Electroporation
}

Axel Boese, INKA HealthTec Innovation Laboratory, Otto-von-Guericke-University, Magdeburg + MEDICS GmbH, Magdeburg, Germany, axel.boese@,med.ovgu.de

Hanbal Arif, INKA HealthTec Innovation Laboratory, Otto-von-Guericke-University, Magdeburg, Germany, hanbal.arif@ovgu.de

Uwe Bernd Liehr, University Clinic Magdeburg, Clinic for Urology, Magdeburg, Germany, UweBernd.Liehr@med.ovgu.de

Johann Jakob Wendler, University Clinic Magdeburg, Clinic for Urology, Magdeburg, Germany, johann.wendler@med.ovgu.de

Michael Friebe, INKA HealthTec Innovation Laboratory, Otto-von-Guericke-University, Magdeburg + IDTM GmbH, Recklinghausen, Germany, info@friebelab.org

\section{Introduction}

Irreversible Electroporation (IRE) is a non-thermal tumor ablation therapy applicable for prostate, kidney, or liver cancer. IRE uses ultra-short and high voltage electrical pulses to destroy the cell membrane leading to apoptosis. The principle of IRE requires two or more needle electrodes placed around the target tissue to apply the electric fields. A pre-requirement to achieve effective ablation is an accurate and parallel needle placement to cover the tumor zone. Differences in tissue density, organ surface curvature, patient motion in combination with long and highly flexible needle electrodes complexify the needle placement. We propose a concept to increase accuracy in IRE needle placement.

\section{Methods}

An analysis of the medical workflow and comparable applications was performed. Additionally, the tissue/needle behavior while placement was studied. Based on the findings, we decided to use a $20 \mathrm{~mm}$ template known from biopsy procedures to improve needle guidance combined with a newly designed shooting mechanism. This shooter allows the insertion of the standard IRE needles and can push the electrode fast up to 40mm into the tissue. This concept uses the inertness of the tissue to achieve a more straight trajectory.

A phantom setup was designed to test the hypothesis. Potato and sausage were embedded into gelatine to mimic a target structure like the prostate. Manual, template-guided, and template-guided shooting placement was performed under U1trasound imaging to analyze the behavior of needle deflection.

\section{Results}

Two hundred punctures in different angles to the target structure surface were performed, and needle deflection was measured in US images. Deflection errors were significantly minimized when the needle was placed with a guidance template and even more combined with the shooting device.

\section{Conclusion}

The parallelity of flexible needles can be improved by applying a more extended guidance template and shooting mechanism similar to biopsy guns. 


\section{Ultrasound Thermometry for HIFU Therapy}

Rosa Daschner, Holger Hewener, Wolfgang Bost, Steffen Weber, Steffen Tretbar, Marc Fournelle

Department of Ultrasound, Fraunhofer-Institut für Biomedizinische Technik (IBMT), Sankt Ingbert, Saarland, 66386

\section{Introduction}

High-Intensity Focused Ultrasound (HIFU) has emerged as an alternative tumour therapy with the ability for selective and notably noninvasive thermal ablation of cancerous tissue. For a safe application, the heat deposition needs to be monitored in real time, which is currently done clinically with Magnetic Resonance imaging. Ultrasound (US) is a promising alternative for therapy monitoring, as it is less expensive and allows the use of a single device for both, therapy and monitoring. In this paper, we present a method for spatial and temporal US thermometry including invitro validation results.

\section{Methods}

The presented method is based on the approximately linear dependence between temperature and speed of sound (SoS) in tissue. By tracking the speckles of successive B-images, the possibility of detecting local changes in SoS and therefore in temperature is given. In this work, a speckle tracking algorithm was implemented for 2D and 3D US Thermometry using a Spatial Compound method to reduce artifacts. The algorithm was initially tested via computational modelling. Afterwards, the technique was experimentally validated in a tissue mimicking gelatine-based phantom and in porcine tissue for temperature rises up to $\triangle 8^{\circ} \mathrm{C}$. We used a curved single element US transducer as therapeutic probe, a linear(/matrix array) transducer with $128(/ 32 \times 32)$ elements for imaging and thermocouples for validation in the $2 \mathrm{D}$ case. To obtain quantitative temperature estimations, independent calibration experiments were conducted.

\section{Results}

In all experiments, both computational and in vitro, we succeeded in locating and monitoring the thermal induced SoS changes over time. The in vitro measurements were in good agreement with the simulation results and the thermocouple measurements $\left(\mathrm{rms}\right.$ temperature difference $=0.44^{\circ} \mathrm{C}$, rms correlation coefficient $\left.=0.95\right)$.

\section{Conclusion}

In this work, we developed a speckle tracking method, that enables the realisation of US Thermometry during HIFU and tested it successfully in invitro experiments. 


\section{Differentiation of Mild Cognitive Impairment Conditions in MR Images using Fractional order Jacobi Fourier Moment Features}

Ravi Dadsena, Research Fellow (Department of Applied Mechanics, Indian Institute of Technology Madras), Chennai, India, ravidadsena34@gmail.com

Deboleena Sadhukhan, Research Fellow (Department of Applied Mechanics, Indian Institute of Technology Madras), Chennai, India, deboleena.rainbow@gmail.com

Ramakrishnan Swaminathan, Professor (Department of Applied Mechanics, Indian Institute of Technology Madras), Chennai, India, sramki@iitm.ac.in

\section{Introduction}

Mild Cognitive Impairment (MCI) is the asymptomatic, preclinical transitional stage among aging and Alzheimer's Disease (AD). Detection of MCI can ensure the timely intervention required to manage the disease's severity. Morphological alterations of Lateral Ventricle (LV) is considered as a significant biomarker for disease diagnosis. This research aims to analyse the shape alterations of the LV region using Fractional Order Jacobi Fourier Moment (FOJFM) features, which are characterized by its generic nature and time-frequency analysis capability.

\section{Methods}

T1-weighted transaxial view brain MR images $(\mathrm{HC}=92$ and $\mathrm{MCI}=63)$ are obtained from publicly available Open Access Series of Imaging Studies (OASIS) database. The LV region is delineated using Weighted Level Set Evolution (WLSE) segmentation method and results are validated against Ground Truth (GT) images. FOJFM features are employed to characterize the morphometry of LV region. From this segmented region, 200 features are computed by varying the value of order and fractional parameters. Support Vector Machine (SVM) and Random Forest (RF) classifiers are used to differentiate $\mathrm{HC}$ and MCI subjects.

\section{Results}

Results shows that, WLSE is able to delineate the LV structure. The segmented region shows good correlation with the GT area. FOJFM shape features are found to be statistically significant in discriminating HC and MCI subjects with $\mathrm{p}<0.05$. For MCI subjects, the feature values show higher variation as compared with HC brain, which might be due to the surface expansion of ventricular area during disease progression. SVM and RF classifiers show high performance Fmeasure value of $93.14 \%$ and $86.24 \%$ respectively for differentiating MCI condition.

\section{Conclusion}

The proposed moment based FOJFM features are able to capture the morphological changes of LV region related to MCI condition. Hence the proposed pipeline of work can be useful for the automated and early diagnosis of diseased conditions. 


\section{Development of a virtual reality simulation for exposure based addiction therapy}

Moritz M. R. Faust*, Department of Applied Sciences and Mechatronics, University of Applied Science Munich, Munich, faust@hm.edu

Christian Hanshans, Department of Applied Sciences and Mechatronics, University of Applied Science Munich, Munich, christian.hanshans@hm.edu

Lukas M. Bröll, Department of Applied Sciences and Mechatronics, University of Applied Science Munich, Munich, $\underline{\text { 1.broell@hm.edu }}$

Bettina Maisch, Strascheg Center for Entrepreneurship, Department of Qualification and Education, University of Applied Science Munich, Munich, bettina.maisch@sce.de

* Corresponding author

\section{Introduction}

After the success of virtual reality (VR) applications in supporting exposure-based and psychoeducational cognitive behavioral therapy for various mental illnesses, the technology is also gaining traction in addiction medicine. This paper will describe the concept behind a custom-developed VR simulation for this application with a particular emphasis on alcohol-related addictive disorders.

\section{Methods}

Using Unreal Engine 4, an exposure simulation and a scenario for learning and applying coping strategies were developed for the Oculus Quest. Because of the common association of supermarkets with the procurement of alcohol among addicts, a small shop was implemented as initial scenario. Since the feeling of presence is an important parameter in the quality assessment of a simulation, the degree of immersion was evaluated in initial practical tests after development.

\section{Results}

In surveys with ten healthy testers, the majority showed a feeling of immersion. Cybersickness and unrealistic collision physics were identified as disruptive factors to the extent of immersion. This work provides the basis for a novel concept for addiction therapy which focusses on a fusion of playful psychoeducation, adjustable exposure, and the internalization of coping strategies with the help of multisensory biofeedback as well as the documentation of the objective physiological measurements for assessment of therapy success.

\section{Conclusion}

The aim is to best possible address the specific requirements for clinical and home use in terms of self-containment, immersion, and ease of use. To evaluate and exploit the scientific potential of this concept scientific studies and technical improvements to both simulation and hardware are required. 


\section{EMG Synchronized Reflex Hammer for precise Patella reflex test}

Óskar Pilkington, Health Technology Center, Reykjavik University/ Landspitali -Uni. Hospital, Reykjavik, Iceland, oskarp@ru.is

Halldór Kárason, Health Technology Center, Reykjavik University/ Landspitali -Uni. Hospital, Reykjavik, Iceland, halldork15@ru.is

Pórður Helgason, Health Technology Center, Reykjavik University/ Landspitali -Uni. Hospital, Reykjavik, Iceland, thordur@landspitali.is

\section{Introduction}

The reflex hammer is a tried and tested method of assessing the health of a patient's nervous system. Its widespread use over the last centuries is in part a result of its simplicity to administer.

However, the use of a standard reflex hammer to test a patients patella reflex only gives the practitioner qualitative information on the patients health whereas to acquire good quantitative data has traditionally required a significantly more complex setup.

\section{Methods}

To gain greater quantitative data on a patient's muscular reflex, an EMG system is used to measure a patient's response to a patellar tendon test. For this purpouse a reflex hammer which includes an accelerometer to accurately measure the force and timing administered by the reflex hammer on a patient's tendon has been developed and its signals syncroniced to the EMG recordings. The system is tested on 12 young volunteers. Between 98 and up to 220 tests where done on each volunteer. The amount of data enables the use of machine learning for the evaluation of the results.

\section{Results}

The Reflex hammer specified above has been designed to work with a wireless EMG system (Kiso ehf). By using wireless EMG system, the administration of this test is simple and fast, requiring no more time than a usual reflext test. No technical adjustments are required by the user. The accelerometer is synchronized with the EMG measurements with an error $<0.6 \mathrm{~ms}$. The impact of the hammer on the patella tendon is quantiatively measured and timed. The intensity and time path of the reflex curve is recorded. Thus the time periods of signal transfer through the monosynaptic reflex arc and the intensity and duration of the EMG answer is measured. The 1700 measurements on healthy volunteers give a good reference data base.

\section{Conclusion}

This device shows great promise to be a quick method of non-invasively quantifying the recovery of patients with a spinal cord injury or brain insult which cause spastic tendancies. 


\title{
Deep brain stimulation related artifacts measured in a Yokogawa-type MEG using a thorax-head saline phantom
}

\author{
Roxanne Lofredi, Charité Universitätsmedizin Berlin, Berlin, Germany, \\ roxanne.lofredi@charite.de \\ Tilmann H. Sander, Physikalisch-Technische Bundesanstalt, Berlin, Germany, \\ tilmann.sander-thoemmes@ptb.de \\ Ahmet L. Kandemir, Heinrich-Heine-University, Medical Faculty Düsseldorf, Germany, \\ ahmet.kandemir@uni-duesseldorf.de \\ Esther Florin, Heinrich-Heine-University, Medical Faculty Düsseldorf, Germany, \\ esther.florin@uni-duesseldorf.de \\ Alfons Schnitzler, Heinrich-Heine-University, Medical Faculty, Düsseldorf, Germany, \\ alfons.schnitzler@uni-duesseldorf.de \\ Andrea Kühn, Charité Universitätsmedizin Berlin, Berlin, Germany, \\ andrea.kuehn@,charite.de \\ Bahne H. Bahners, Heinrich-Heine-University, Medical Faculty Düsseldorf, Germany, \\ bahne.bahners@uni-duesseldorf.de
}

\section{Introduction}

Deep brain stimulation (DBS) is an effective therapy for patients with movement disorders. Some of the DBS-effects are thought to rely on the modulation of distributed network activity. Whole-head magnetoencephalography (MEG)-recordings during DBS can be used to investigate those effects. However, when conducting MEG-DBS recordings, strong artifacts have to be considered.

\section{Method}

Two boreholes were drilled in a plastic mannequin for bilateral insertion of DBS-electrodes. Flexible inserts were manufactured by 3D printing to get watertight plugs with feedthroughs for the electrodes of type Medtronic 3389. The phantom was filled with a saline solution. An electric test dipole was placed shallow in the phantom to mimic a cortical current source. Both DBS electrodes were used with bipolar montage, a pulse width of $90 \mu \mathrm{s}$ and a frequency of $130 \mathrm{~Hz}$. During some recordings the phantom was moved to simulate movement artifacts and a reference condition without stimulation was recorded. The MEG signal was sampled at $10 \mathrm{kHz}$ with a bandwidth from $0.3-5 \mathrm{kHz}$ and gain was reduced from normal settings to avoid saturation. Artifact contamination was assessed by calculating the root mean squared error (RMSE) of the logarithmic power between reference recording and DBS recordings.

\section{Results}

Two types of artifacts were observed. Firstly, rectangular-shaped DBS pulses result in a contamination of the Welch power spectrum with harmonics of the stimulation frequency. For the $130 \mathrm{~Hz}$ peak, the RMSE was 0.104 ( $3 \mathrm{~mA})$ and $0.152(6.5 \mathrm{~V})$ respectively. Secondly, the movement of ferromagnetic DBS hardware components led to low-frequency artifacts - mainly in the left-sided sensors (RMSE between 1 and $15 \mathrm{~Hz}$ : 0.120). Using a combination of independent component analysis (ICA) and spectral signal space projection (S3P) the movement and DBS pulse artifacts could be reduced.

\section{Conclusion}

The Yokogawa MEG can record probe dipole signals in a saline thorax-head phantom while a Medtronic stimulator delivers biphasic rectangular-shaped pulses to electrodes in the phantom. This indicates suitability for combined MEGDBS studies in movement disorder patients. Future studies have to investigate the applicability of DBS artifact reduction methods for Yokogawa recordings. 